SHORT REPORT

\title{
Is variant Creutzfeldt-Jakob disease in young children misdiagnosed as Alpers' syndrome? An analysis of a national surveillance study
}

\author{
J te Water Naudé, C M Verity, R G Will, G Devereux, L Stellitano
}

J Neurol Neurosurg Psychiatry 2004;75:910-913. doi: 10.1136/jnnp.2003.015651

\begin{abstract}
Background: There has been concern that children with variant Creutzfeldt-Jakob disease (vCJD) might be misdiagnosed as cases of Alpers' syndrome, as a spongiform degeneration of the brain is seen in both conditions.

Objective: To report a national prospective surveillance study of children with progressive intellectual and neurological deterioration, designed to detect any children in the United Kingdom with VCJD, to see whether this misdiagnosis is occurring.

Methods: A monthly surveillance card is sent by the British Paediatric Surveillance Unit to all consultant paediatricians in the UK. The card lists the disorders currently under surveillance. Paediatricians are asked to return the card, reporting cases seen in the previous month. The BPSU office informs the surveillance groups about reported cases, and they obtain clinical information from the notifying paediatrician.

Results: After 5 years and 8 months of surveillance, 1244 children had been reported to the study. Alpers' syndrome was confirmed in two, although this was the suggested diagnosis in 11 children at the time of initial notification. Conclusions: The results show that Alpers' syndrome is rare and it is unlikely that vCJD cases are being misdiagnosed as Alpers' syndrome.
\end{abstract}

$\mathrm{N}$ ational prospective surveillance for children with progressive intellectual and neurological deterioration (PIND) was begun through the British Paediatric Surveillance Unit (BPSU) in May 1997. The main aim of the PIND study is to identify any children in the United Kingdom who have developed variant Creutzfeldt-Jakob disease (vCJD). ${ }^{1}$ There was concern that vCJD in children might be misdiagnosed as Alpers' syndrome, for two reasons. First, the neuropathological findings in these two conditions have some similarities. ${ }^{2-4}$ Second, it is reported that a spongiform encephalopathy was produced in hamsters following the inoculation of brain tissue from a young child with Alpers' syndrome. ${ }^{5}$ The neuropathology of the hamster brains was similar to that seen in cases of interspecies transfer of vCJD. ${ }^{6}$

The six reported cases of $\mathrm{vCJD}$ in children developed symptoms between 12 and 15 years of age. ${ }^{17}$ Their presentation was identical to that described in adults. Marked personality and mood change was the initial finding, followed by rapid cognitive decline, with gait disturbance, myoclonus, and death. The neuropathology of vCJD is characteristic with grey matter spongiform degeneration, reactive astrocytic gliosis, and "florid plaques" which are pathognomonic of vCJD. They are composed of a core of
$\mathrm{PrP}^{\mathrm{SC}}$-amyloid surrounded by vacuoles $\left(\mathrm{PrP}^{\mathrm{SC}}\right.$ is the disease causing isoform of the normal cellular prion protein $\left.^{8}\right)$.

Alpers' syndrome (also known as Alpers' disease or progressive neuronal degeneration of childhood) usually starts in children under two years (see table 1). There is psychomotor retardation, intractable seizures (typically myoclonic), cortical blindness, and terminal liver failure. ${ }^{2}$ Electroencephalograms (EEGs) show high amplitude slow activity with polyspikes, visual evoked potentials (VEPs) are abnormal, and cranial imaging shows a progressive brain atrophy, usually occipital. Death occurs six to 12 months after the onset of symptoms. The brain shows patchy cortical astrocytosis, with neuronal loss and spongiform change. ${ }^{9}$ The condition can be inherited in an autosomal recessive fashion, and may be caused by mitochondrial abnormalities. ${ }^{10-13}$

Because numerous reports have mentioned the pathological similarities between vCJD and Alpers' syndrome, ${ }^{2-4}$ we reviewed all cases notified to the PIND study with a suggested diagnosis of Alpers' syndrome.

\section{METHODS}

A monthly surveillance card is sent by the BPSU to all consultant paediatricians in the UK. The card lists the disorders currently under surveillance. Paediatricians are asked to return the card, reporting cases seen in the previous month. The BPSU office informs the surveillance groups about reported cases, and they obtain clinical information from the notifying paediatrician. The full methodology is given in the BPSU annual report. ${ }^{7}$

Using the BPSU system, paediatricians are asked to report children with "progressive intellectual and neurological deterioration" (see panel $\mathrm{l}$ in Verity et al, 2000 ${ }^{1}$ ). An expert group meets quarterly to study the anonymised clinical information and classify cases by diagnosis. The PIND team does not become actively involved with the patient or the family, and investigations are carried out by the paediatricians who are already involved. However, if there are any cases with features suggestive of VCJD (see table $2^{16}$ ) the protocol is for them to be discussed with the local paediatrician with a view to referral to the National CJD Surveillance Unit for further investigations for vCJD.

Ethical consent for the PIND study was obtained from the Cambridge local research ethics committee and from the Public Health Laboratory Service ethics committee.

\section{RESULTS}

Between May 1997 and December 2002, paediatricians reported 1244 children with suspected PIND. In 11 cases the referring paediatrician suggested that the diagnosis might

Abbreviations: BPSU, British Paediatric Surveillance Unit; PIND, progressive intellectual and neurological deterioration; VCJD, variant Creutzfeldt-Jakob disease 
Table 1 Reported clinical findings in Alpers' syndrome 2414

\begin{tabular}{l} 
Clinical course \\
- Normal birth and early development \\
- Slow onset of developmental delay \\
- Hypotonia, often with vomiting and failure to thrive \\
- Seizures, often intractable, before a progressive deterioration \\
- Clinically apparent hepatic dysfunction, usually terminal \\
- Death, usually before the age of three years \\
Investigations may show the following \\
- Liver function tests may be abnormal before the onset of seizures \\
- EEG shows slow activity with high amplitude, mixed with low amplitude high frequency polyspikes \\
- VEP are absent, asymmetrical, or with an ill defined initial component, with a normal electroretinogram ${ }^{2}$ \\
- There is progressive cerebral atrophy on cranial imaging, most marked in the occipital region \\
- Liver pathology shows severe microvesicular fatty change, diffuse bile duct proliferation, bridging fibrosis, a \\
disorganised hepatic architecture, with ongoing hepatocyte necrosis \\
- Macroscopic brain pathology shows a variably distributed thinned, granular, and discoloured cortical ribbon, \\
typically involving the calcarine cortex \\
- Brain pathology typically affects the striate and occipital cortex and ranges from a mild superficial astrocytosis, \\
through an increasingly deep sponginess, nerve cell loss and gliosis, to a thin and disorganised cortical ribbon, \\
with hypertrophic astrocytes replacing nerve cells and a prominent vascular component \\
- Atypical forms exist, with a fulminant course with seizures predominating, a prolonged survival, or late \\
presentations, sometimes with an axonal neuropathy ${ }^{15}$ \\
\hline EEG, electroencephalogram; VEP, visual evoked potentials.
\end{tabular}

be Alpers' syndrome. Common to all was developmental delay with feeding problems. The majority had seizures, of different types. Most had hepatic dysfunction, although overt liver failure was noted in two cases only. Clinical details are shown in table 3.

The PIND study expert group agreed that two cases met the criteria for Alpers' syndrome (table 1). The age at onset of the first case (table 3, case 1) was 19 months, with failure to thrive, developmental delay, microcephaly, and hypotonia. There were no seizures initially, but generalised seizures later. The course was progressive, with death at 23 months. Liver dysfunction was present, with an abnormal but not characteristic EEG. Brain and liver biopsies were characteristic of Alpers' syndrome (table 1).

The second child with a diagnosis of Alpers' syndrome (table 3, case 2) presented with an aggressive seizure disorder aged eight months. The seizures were initially generalised, and then became myoclonic. This child was hypotonic but not microcephalic and there was no developmental delay before the onset of seizures. VEPs were asymmetrical, and an EEG showed a burst-suppression pattern. Death was caused by liver failure at age of 10 months. Liver histology showed microvesicular fat, minimal fibrosis, and portal tract expansion, consistent with an Alpers'-like disease or a mitochondrial defect. The expert group thought that the clinical features were acceptable for the diagnosis of Alpers' syndrome, even though brain histology was not available.

In the remaining cases the diagnosis was not thought to be Alpers' syndrome. The expert group thought that cases 3, 4, and 5 had mitochondrial cytopathies. Cases 3 and 4 had myoclonic seizures as part of their initial presentation. EEGs were abnormal but not typical of Alpers' syndrome. In case 3 there was hypotonia and a raised CSF lactate. Postmortem muscle and liver biopsies were consistent with a mitochondrial cytopathy, with type II atrophy and negative cytochrome oxidase fibres present in muscle, and mild periportal fibrosis in the liver. Case 4 had an acute presentation with rhabdomyolysis, acute renal failure, hypoglycaemia, a coagulopathy, and focal seizures. Clinically there was microcephaly and hypotonia, and raised CSF lactate. Cranial magnetic resonance imaging (MRI) showed basal ganglia changes typical of a mitochondrial cytopathy. Visual evoked

Table 2 Diagnostic criteria for variant CJD

\begin{tabular}{|c|c|}
\hline I & $\begin{array}{l}\text { A: Progressive neuropsychiatric disorder } \\
\text { B: Duration of illness more than six months } \\
\text { C: Routine investigations do not suggest an alternative diagnosis } \\
\text { D: No history of potential iatrogenic exposure }\end{array}$ \\
\hline II & $\begin{array}{l}\text { A: Early psychiatric symptoms* } \\
\text { B: Persistent painful sensory symptoms } \dagger \\
\text { C: Ataxia } \\
\text { D: Myoclonus, chorea, or dystonia } \\
\text { E: Dementia }\end{array}$ \\
\hline III & $\begin{array}{l}\text { A: EEG does not show typical appearance of sporadic CJD } \ddagger \text { (or no EEG done) } \\
\text { B: Bilateral pulvinar high signal on magnetic resonance imaging }\end{array}$ \\
\hline $\begin{array}{l}\text { Definite vCJD } \\
\text { Probable vCJD } \\
\text { Possible vCJD }\end{array}$ & $\begin{array}{l}\text { IA (progressive neuropsychiatric disorder) and neuropathological confirmation of vCJD§ } \\
\text { I and } 4 / 5 \text { of II and IIIA and IIIB } \\
\text { I and } 4 / 5 \text { of II and IIIA }\end{array}$ \\
\hline \multicolumn{2}{|c|}{$\begin{array}{l}\text { *Depression, anxiety, apathy, withdrawal, delusions. } \\
\text { †This includes both frank pain, unpleasant dysaesthesia, or both. } \\
\text { †Generalised triphasic complexes at about } 1 \mathrm{~Hz} \text {. } \\
\text { §Spongiform change and extensive prion protein deposition with florid plaques throughout cerebrum and } \\
\text { cerebellum. } \\
\text { EEG electroencephalogram; vCJD, variant Creutzfeldt-Jakob disease. }\end{array}$} \\
\hline
\end{tabular}




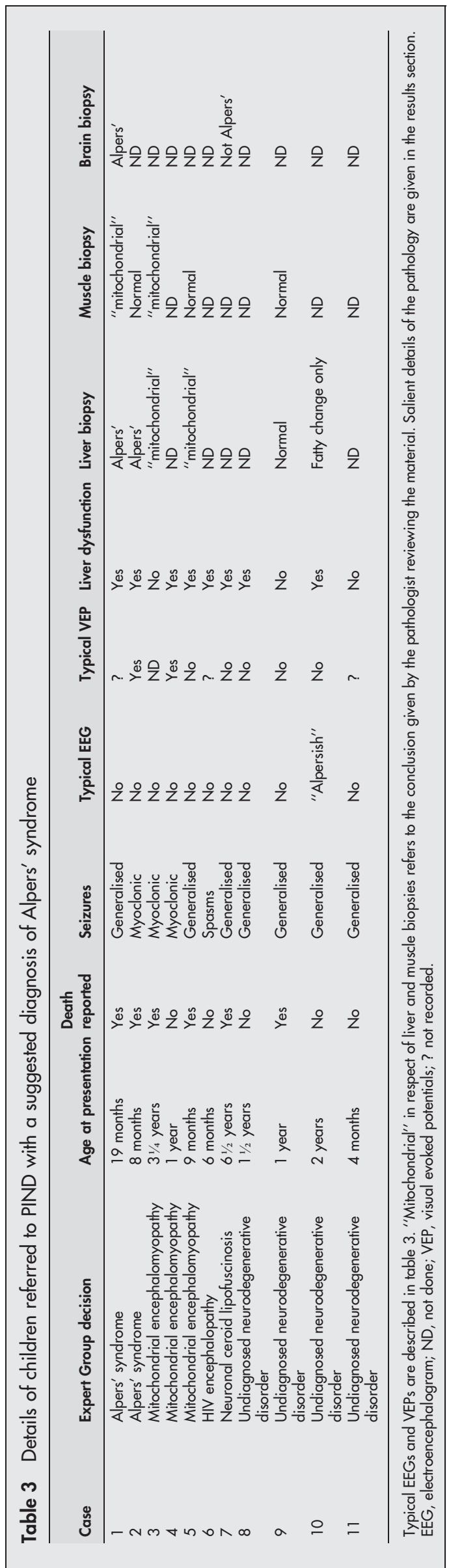

potentials (VEPs) were absent, with normal electroretinograms (ERGs). There was evidence of liver dysfunction, but no biopsies were done. Case 5 presented in status epilepticus and died two months later of liver failure. Liver biopsy showed microvesicular steatosis with early fibrosis. This child had severely depleted levels of mitochondrial DNA in the liver.

Case 6 presented at six months with developmental delay, possible seizures, hypotonia, and liver dysfunction. Further serological and DNA investigations confirmed that the diagnosis was HIV encephalopathy. Case 7 presented aged $6 \frac{1}{2}$ years with generalised tonic-clonic seizures, developmental delay, and hypotonicity. The rectal biopsy suggested neuronal ceroid lipofuscinosis type 2 (NCL2) because of "fingerprint" inclusions. An ERG was extinguished. This child died aged nine years. The age and relatively slow rate of deterioration were not typical of Alpers' syndrome.

Cases 8, 9, 10, and 11 had undiagnosed neurodegenerative disorders. None had VEPs suggestive of Alpers' syndrome. Case 8 had generalised seizures with microcephaly and hypertonicity. An EEG was non-specifically abnormal and cranial MRI showed global cortical atrophy. In case 9 there was hypotonia with a raised plasma ammonia. Liver function was normal. The MRI showed dysmyelination. Case 10 had generalised seizures with hypertonia. The EEG showed spikewave activity and there was occipital atrophy on MRI. Liver biopsy showed mild fatty change only, not typical of Alpers' syndrome. In case 11 there was rapid deterioration with seizures at the age of four months. There was evidence for a congenital cytomegalovirus infection on the basis of serological testing and brain imaging, but this was not thought sufficient to explain the clinical picture. Other investigations did not confirm Alpers' syndrome: there was no abnormality of liver function, the EEG was not abnormal, and cranial MRI showed reduced white matter bulk only.

\section{DISCUSSION}

The aim of the PIND study is to identify any children under 16 years of age in the United Kingdom who develop vCJD. ${ }^{16}$ Confirmation of a diagnosis of vCJD still relies on neuropathological study of the brain. ${ }^{14}$ The strategy of the PIND study is to gather as much clinical and pathological information as is available for each notified child. ${ }^{1}$ For all these children the PIND study expert group looks for evidence of a clinical presentation similar to that already described for vCJD. ${ }^{14}$ The aim is to ensure that any such children are referred to the National CJD Unit in Edinburgh for full investigation after consent is obtained. However, there remains the possibility that some young children with vCJD might present with a disease picture that is not similar to that described in older patients.

Only a small proportion of the children in the PIND study who died underwent brain biopsy or necropsy examination, which reflects current UK practice. Fortunately the majority of children with PIND are found by other tests to have a diagnosis that is not vCJD. For instance after three years of surveillance the group of 360 diagnosed cases of PIND was made up of 88 different known neurodegenerative diseases. However, at that time 30 children had no diagnosis because they had either died (13 cases) or had been investigated without success ( 17 cases). These 30 children were clinically heterogeneous and among them there was no evidence of a new clinical syndrome that might be a previously unrecognised type of CJD. ${ }^{1}$

A particular concern was that some young children with vCJD might be misdiagnosed as Alpers' syndrome (see above). It is therefore reassuring that after five years and eight months of active national surveillance the PIND study expert group concluded that there were only two cases of 
Alpers' syndrome and in one of those brain histology confirmed that diagnosis, rather than vCJD. In neither of the two cases were there any clinical features suspicious of vCJD. In nine other cases the local paediatricians had initially suggested a diagnosis of Alpers' syndrome, but the expert group did not agree, sometimes because subsequent investigations suggested other diagnoses.

Unfortunately brain histology is available in only small proportion of children who die. Without histology it is not possible to exclude vCJD completely. However, our study reassures us that children with PIND are being carefully investigated by their local paediatricians and tertiary specialists. This paper shows how the PIND study follows children with suspicious clinical findings. Initially 11 children were thought to have Alpers' syndrome. They were kept under surveillance while local investigations proceeded and were reclassified as shown in table 3.

It seems probable that an increasing number of children previously categorised as Alpers' syndrome will be diagnosed as having a specific mitochondrial cytopathy. ${ }^{10-13}$ Our population based study shows that Alpers' syndrome is very rare and that it is unlikely that cases of VCJD in children are being misdiagnosed as Alpers' syndrome, despite some concern that necropsy material is rarely available after children die of neurodegenerative disease.

\section{ACKNOWLEDGEMENTS}

The PIND study is funded by the Department of Health. Many thanks to the British Paediatric Surveillance Unit of the Royal College of Paediatrics and Child Health and to Mr Richard Lynn, the scientific coordinator. The paediatric neurologists in the expert group are an invaluable resource. They are: Professor Jean Aicardi, Dr Peter Baxter, Dr Stuart Green, Professor Richard Robinson, Dr Robert Surtees, and Dr John Wilson. This study could not have been done without the hundreds of paediatricians who have reported cases, completed forms, and undergone telephone interviews.

\author{
Authors' affiliations \\ J te Water Naudé, C M Verity, G Devereux, L Stellitano, PIND \\ Surveillance Group, Addenbrooke's Hospital, Cambridge, UK \\ R G Will, National Creutzfeldt-Jakob Disease Surveillance Unit, Western \\ General Hospital, Edinburgh, UK \\ Competing interests: none declared
}

Correspondence to: Dr Christopher Verity, Child Development Centre, Addenbrooke's Hospital, Hills Road, Cambridge CB2 2QQ, UK; christopher.verity@addenbrookes.nhs.uk

Received 28 March 2003

In revised form 23 July 2003

Accepted 9 September 2003

\section{REFERENCES}

1 Verity CM, Nicoll A, Will RG, et al. Variant Creutzfeldt-Jakob disease in UK children: a national surveillance study. Lancet 2000;356:1224-7.

2 Harding BN. Progressive neuronal degeneration of childhood with liver disease (Alpers-Huttenlocher syndrome): a personal review. J Child Neurol 1990;5:273-87.

3 Crompton MR. Alpers' disease - a variant of Creutzfeldt-Jakob disease and subacute spongiform encephalopathy? Acta Neuropathol (Berl) 1968;10:99-104.

4 Harding BN, Egger J, Portmann B, et al. Progressive neuronal degeneration of childhood with liver disease. Brain 1986;109:181-206.

5 Manuelidis EE, Rorke LB. Transmission of Alpers' disease (chronic progressive encephalopathy) produces experimental Creutzfeldt-Jakob disease in hamsters. Neurology 1989:39:615-21.

6 Bruce ME, Will RG, Ironside JW, et al. Transmissions to mice indicate that "new variant" CJD is caused by the BSE agent. Nature 1977;389:498-501.

7 British Paediatric Surveillance Unit. Sixteenth annual report 2001-2002. London: Royal College of Paediatrics and Child Health, 2002

8 Prusiner SB. Shattuck lecture - Neurodegenerative diseases and prions. N Engl J Med 2001;344:1516-26.

9 Harding BN, Alsanjari N, Smith SJ, et al. Progressive neuronal degeneration of childhood with liver disease (Alpers' disease) presenting in young adults. J Neurol Neurosurg Psychiatry 1995;58:320-5.

10 Naviaux RK, Nyhan WL, Barshop BA, et al. Mitochondrial DNA polymerase gamma deficiency and mtDNA depletion in a child with Alpers' syndrome. Ann Neurol 1999;45:54-8.

11 Rasmussen M, Sanengen T, Skullerud K, et al. Evidence that AlpersHuttenlocher syndrome could be a mitochondrial disease. J Child Neurol 2000;15:473-7.

12 Gauthier-Villars M, Landrieu P, Cormier-Daire V, et al. Respiratory chain deficiency in Alpers syndrome. Neuropediatrics 2001;32:150-2.

13 Uusimaa J, Finnila S, Vainionpaa L, et al. A mutation in mitochondrial DNAencoded cytochrome c oxidase II gene in a child with Alpers-Huttenlocher-like disease. Pediatrics 2003;111:e262-8.

14 Ellison D, Love S, Chimelli L, et al. Neuropathology. London: CV Mosby, 1998 .

15 Simonati A, Filosto M, Tomelleri G, et al. Central-peripheral sensory axonopathy in a juvenile case of Alpers-Huttenlocher disease. J Neurol 2003;250:702-6.

16 Will RG, Zeidler M, Stewart GE, et al. Diagnosis of new variant CreutzfeldtJakob disease. Ann Neurol 2000;47:575-82. 